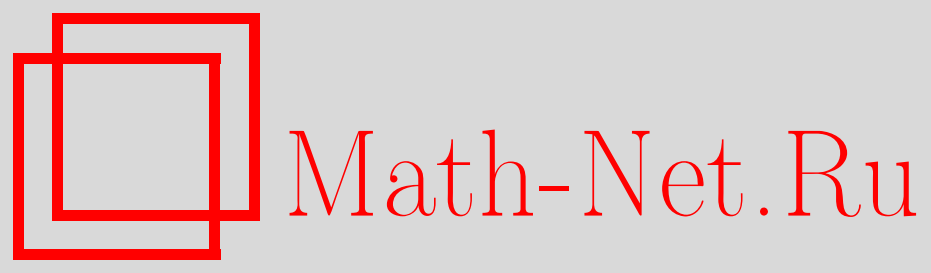

М. Дидин, П. Кожевников, Педальные отрезки: где максимум?, Квант, 2021, номер 6, 18-22

DOI: https://doi.org/10.4213/kvant20210603

Использование Общероссийского математического портала Math-Net.Ru подразумевает, что вы прочитали и согласны с пользовательским соглашением http://www . mathnet.ru/rus/agreement

Параметры загрузки:

IP: 54.164 .48 .24

26 апреля 2023 г., 09:28:59

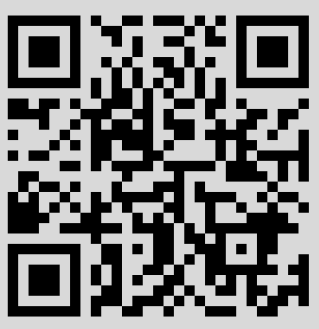




\section{Педальные отрезки: где максимум?}

\section{М.ДИДИН, П.КОЖЕВНИКОВ}

Пусть на плоскости дан треугольник $A B C$ и точка $X$. Проекции точки $X$ на стороны $B C, C A, A B$ обозначим соответственно $X_{a}, X_{b}, X_{c}$. Как известно, точки $X_{a}, X_{b}, X_{c}$ будут лежать на одной прямой тогда и только тогда, когда $X$ лежит на описанной окружности треугольника $A B C$ (в таком случае прямая $X_{a} X_{b} X_{c}$ называется прямой Симсона). Далее считаем, что $X$ не лежит на окружности $(A B C)$, тогда проведем через точки $X_{a}, X_{b}, X_{c}$ окружность - так называемую педальную окружность точки $X$ (рис.1). Вторые точки пересечения окружности $X_{a} X_{b} X_{c}$ со сторонами треугольника обозначим $Y_{a}, Y_{b}, Y_{c}$. Педальными отрезками точки $X$ назовем отрезки $X_{a} Y_{a}, X_{b} Y_{b}, X_{c} Y_{c}$.

В этой небольшой статье мы изучим, какой из педальных отрезков будет макси-

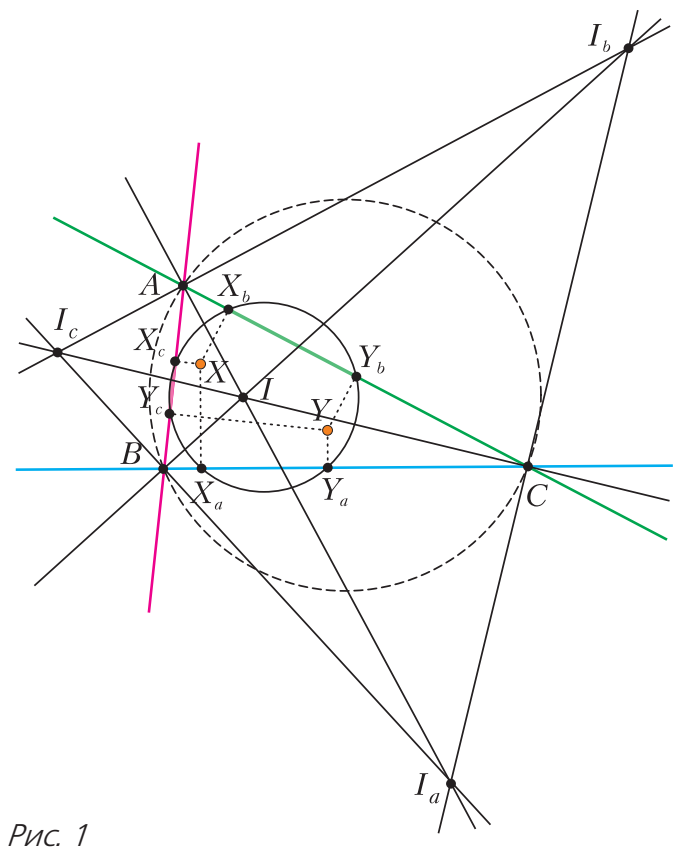

DOI: https://doi.org/10.4213/kvant20210603 мальным или минимальным по длине, в зависимости от положения точки $X$. По результатам этого небольшого исследования мы рассмотрим некоторые интересные частные случаи и дадим решение задачи M2621 из «Задачника «Кванта» (см. «Квант» №9 за 2020 г.). Авторское решение этой задачи опиралось на вычисление длин педальных отрезков.

Будем использовать следующий известный факт (см., например, статью П.Кожевникова «Изогонально сопряженные точки» в «Кванте» № 1 за 2016 г. ): перпендикуляры к прямым $B C, C A, A B$, проведенные в точках $Y_{a}, Y_{b}, Y_{c}$ соответственно, всегда пересекутся в одной точке $Y$, изогонально сопряженной точке $X$, т.е. в точке $Y$ такой, что прямые $A X$ и $A Y$ симметричны относительно биссектрисы угла $B A C$, прямые $B X$ и $B Y$ симметричны относительно биссектрисы угла $A B C$, прямые $C X$ и $C Y$ симметричны относительно биссектрисы угла $A C B$. Иначе говоря, точки $X$ и $Y$ в наших рассмотрениях равноправны, они имеют общую педальную окружность. Точке $Y$ мы также запретим лежать на окружности $(A B C)$ (в этом случае $X$ не определена, или, как говорят, «ушла в бесконечность»), кроме случая, когда $X$ лежит на одной из прямых $A B, B C, C A-$ тогда педальная окружность точки $X$ определена и проходит через вершину треугольника $A B C$, а $Y$ совпадает с этой вершиной.

Основная идея будет в следующем. Мы найдем геометрическое место точек $X$, для которых найдутся два равных педальных отрезка, это ГМТ окажется объединением нескольких прямых и окружностей, которые разобьют плоскость на области. Далее позволим точке $X$ непрерывно двигаться по плоскости, не пересекая «критической окружности» $(A B C)$; при этом длины педальных отрезков будут меняться непрерывно, а значит, пока $X$ находится внутри одной из определенных выше областей, упорядоченность по длине педальных отрезков $X_{a} Y_{a}, X_{b} Y_{b}, X_{c} Y_{c}$ сохранится. Далее проведем внутренние и внешние биссектрисы треугольника; пусть точки их 
пересечения - $I, I_{a}, I_{b}, I_{c}$ - центры вписанной и вневписанных окружностей. Отметим, что $I, I_{a}, I_{b}, I_{c}$ - это в точности точки, совпадающие со своими изогонально сопряженными; можно сказать, что для этих точек длины всех педальных отрезков равны 0.

Будем рассматривать точки $X$, отличные от $I, I_{a}, I_{b}, I_{c}$, с условием $X_{b} Y_{b}=X_{c} Y_{c}$. Проекции $X_{b} Y_{b}$ и $X_{c} Y_{c}$ отрезка $X Y$ на прямые $A C$ и $A B$ равны по длине тогда и только тогда, когда $X Y$ составляет равные углы с прямыми $A C$ и $A B$, т.е. когда отрезок $X Y$ параллелен либо внутренней, либо внешней биссектрисе угла $B A C$.

При этом если $X Y \| I_{b} I_{c}$, то (острый) угол между прямыми $X Y$ и $B C$ меньше, чем угол между прямыми $X Y$ и $A C$ (или $A B$ ), поэтому длина проекции отрезка $X Y$ на прямую $B C$ больше длины проекции на прямую $A B$ или $A C$, т.е. $X_{a} Y_{a}>X_{b} Y_{b}=$ $=X_{c} Y_{c}$.

Если же $X Y \| I I_{a}$, то (острый) угол между прямыми $X Y$ и $B C$ больше, чем угол между прямыми $X Y$ и $A C$ (или $A B$ ), значит, в этом случае $X_{a} Y_{a}<X_{b} Y_{b}=X_{c} Y_{c}$.

Лемма. Пусть дан прямоугольный треугольник $P Q R, \angle R=90^{\circ}$, и различные точки $X$ и $Y$, симметричные относительно прямой $P Q$. Прямые $R X$ и $R Y$ симметричны относительно прямой $R P$ тогда и только тогда, когда $X$ и $Y$ лежат на окружности ( $P Q R)$.

Доказательство. Если $X$ и $Y$ лежат на окружности $(P Q R)$ (т.е. окружности, построенной на $P Q$ как на диаметре; рис.2), то дуги $P X$ и $P Y$ равны, поэтому $R P-$ биссектриса (внутренняя либо внешняя) угла $X R Y$.

Пусть, наоборот,

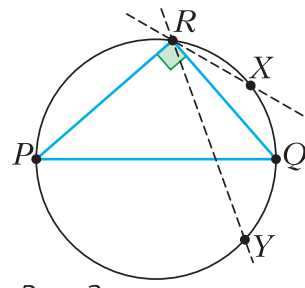

Pис. 2

$R P$ - биссектриса угла между прямыми $R X$ и $R Y$. Тогда $R P$ пересекает серединный перпендикуляр $Q P$ к отрезку $X Y$ в серединах двух дуг $X Y$ окружности $(R X Y)$. Но эти точки пересечения $-P$ и $Q$, поэтому все пять точек $R, P, Q, X, Y$ лежат на одной окружности. Лемма доказана.
Рассмотрим условие $X Y \| I I_{a}$. Это условие, очевидно, выполнено, когда $X$ лежит на самой биссектрисе $I I_{a}$ угла $A$. Пусть далее $X$ не лежит на прямой $I I_{a}$. Тогда прямые $A X$ и $A Y$ не совпадают и симметричны относительно биссектрисы (внешней и внутренней) угла $A$, а поскольку $X Y \| I I_{a}$, точки $X$ и $Y$ должны быть симметричны относительно внешней биссектрисы $I_{b} I_{c}$ угла $A$. Но тогда для точек $X, Y$ и прямоугольных треугольников $I_{b} I_{c} B$, $I_{b} I_{c} C$ выполнены условия леммы, а значит, $X$ и $Y$ лежат на окружности $\left(I_{b} I_{c} B C\right)$ (с диаметром $I_{b} I_{c}$ ). Наоборот, если $X$ и $Y$ лежат на этой окружности (и симметричны относительно $I_{b} I_{c}$ ), то $X$ и $Y$ изогонально сопряжены. Итак, мы получили такое утверждение.

Утверждение 1. Условие $X_{a} Y_{a}<X_{b} Y_{b}=$ $=X_{c} Y_{c}$ выполнено тогда и только тогда, когда $X$ лежит на прямой $I I_{a}$ или на окружности $\left(I_{b} I_{c} B C\right)$.

\section{Диаграмма максимумов}

Будем следить за максимальным из трех педальных отрезков. Проведем все три биссектрисы и окружности $\left(I_{b} I_{c} B C\right)$, $\left(I_{c} I_{a} C A\right),\left(I_{a} I_{b} A B\right)$ (рис.3). Проведенные прямые и окружности разбивают плоскость на области. Оказывается (и сейчас мы это покажем), что максимум из трех педальных отрезков определяется цветом области, в которой лежит точка $X$ : если $X$ лежит в голубой области, то максималь-

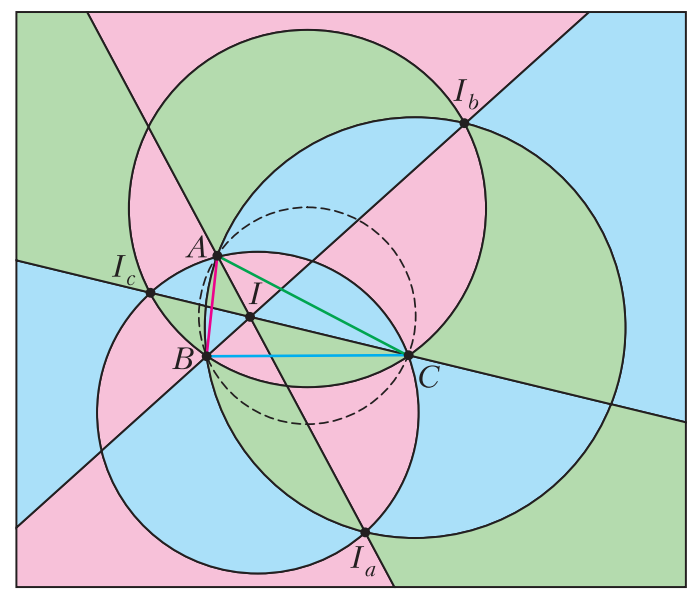

Pnc. 3 
ным является педальный отрезок $X_{a} Y_{a}$, если в зеленой - то $X_{b} Y_{b}$, а если в розовой то $X_{c} Y_{c}$.

Пусть для точек $X$ из некоторой области максимальным педальным отрезком является $X_{b} Y_{b}$. Будем непрерывно двигать точку $X$, не пересекая «критической» окружности $(A B C)$. Пусть $X$ перешла из одной области в соседнюю (граничащую по отрезку или дуге) область; такой переход будем называть «катастрофой». Из утверждения 1 следует, что после катастрофы максимальным мог стать отрезок $X_{c} Y_{c}$ только в случае перехода точки $X$ через прямую $I I_{a}$ либо через окружность $\left(I_{b} I_{c} B C\right)$. После такого перехода проекция вектора $\overrightarrow{X Y}$ на внешнюю биссектрису меняет направление (покажите это!), поэтому действительно максимальный отрезок меняется с $X_{b} Y_{b}$ на $X_{c} Y_{c}$.

Несложно определить цвет некоторых областей. Например, если $X$ находится вблизи отрезка $A I$ внутри треугольника, образованного прямыми $A B, A I, C I$, то $Y$ находится вблизи прямой $A I$ внутри треугольника, образованного прямыми $B C, A I$, $C I$; в таком случае направление $\overrightarrow{X Y}$ близко к направлению $\overrightarrow{A I}$, но (острый) угол между $\overrightarrow{X Y}$ и $\overrightarrow{A B}$ больше, чем (острый) угол между $\overrightarrow{X Y}$ и $\overrightarrow{A C}$, поэтому для таких точек $X$ (и $Y$ ) верно $X_{a} Y_{a}<X_{c} Y_{c}<X_{b} Y_{b}$ и соответствующие области на рисунке 3 (криволинейные треугольники, образованные прямыми $A I, C I$ и одной из дуг окружности $\left(I_{a} I_{b} A B\right)$ или $\left.\left(I_{b} I_{c} B C\right)\right)$ должны быть зелеными. Далее цвет каждой из областей, лежащих внутри окружности $(A B C)$, можно определить, отслеживая катастрофы при переходе из одной области в соседнюю.

Пусть теперь $X$ находится вне окружности $(A B C)$, скажем, $X$ лежит в области, расположенной непосредственно над дугой $A I_{b}$. Тогда нетрудно видеть, что $Y$ лежит внутри окружности $(A B C)$ в области, про которую уже известно, что она зеленая. Значит, область, которой принадлежит $X$, тоже зеленая. Определить цвет других областей, лежащих вне окружности $(A B C)$, можно опять, отслеживая ката- строфы. Таким образом получается раскраска на рисунке 3 ; по факту оказывается, что переход через критическую окружность $(A B C)$ не меняет цвет области.

\section{Диаграмма минимумов}

Рассуждения, аналогичные проведенным выше, можно провести и для минимального педального отрезка. Аналогом утверждения 1 будет следующее.

Утверждение 2. Условие $X_{a} Y_{a}>X_{b} Y_{b}=$ $=X_{c} Y_{c}$ выполнено тогда и только тогда, когда $X$ лежит на прямой $I_{b} I_{c}$ или на окружности $\left(I I_{a} B C\right)$.

За минимум отвечает принадлежность точки $X$ какой-то области, на которые плоскость разбивают внешние биссектрисы и окружности $\left(I I_{a} B C\right),\left(I I_{b} C A\right)$, $\left(I I_{c} A B\right)$ (рис.4). Читатель может провести

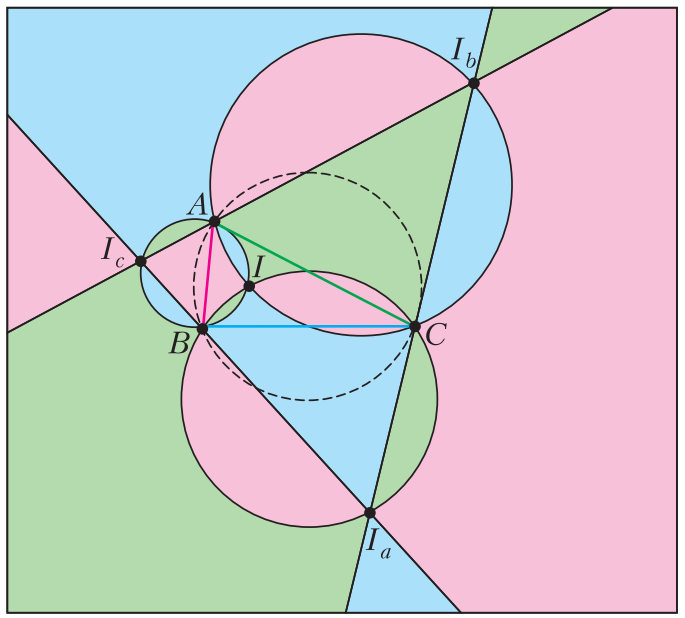

Pис. 4

рассуждения в подробностях и убедиться, что в качестве диаграммы минимумов получается раскраска на рисунке 4.

\section{Решение задачи М2621}

M2621 (И. Вайнштейн). Дан треугольник $A B C$, в котором $A B<B C<C A$. Вневписанные окружности касаются сторон $B C, C A, A B$ в точках $A_{1}, B_{1}$ и $C_{1}$ соответственно. Через точки $A_{1}, B_{1} u C_{1}$ проведена окружность, которая вторично пересекает стороны $B C, C A, A B$ в точках $A_{2}$, $B_{2}$ и $C_{2}$ соответственно. На какой из сторон треугольника может лежать наи больший из отрезков $A_{1} A_{2}, B_{1} B_{2} u C_{1} C_{2}$ ? 
Для решения этой задачи заметим, что перпендикуляры, проведенные к сторонам треугольника через точки касания с вневписанными окружностями, проходят через центры вневписанных окружностей, т.е. это перпендикуляры, проведенные через $I_{a}, I_{b}, I_{c}$ к прямым $B C, C A, A B$ соответственно. Иными словами, это перпендикуляры, опущенные из вершин треугольника $I_{a} I_{b} I_{c}$ на стороны его ортотреугольника. Как известно, они пересекаются в центре $O^{\prime}$ окружности $\left(I_{a} I_{b} I_{c}\right)$. Таким образом, окружность из условия задачи это педальная окружность точки $X=O^{\prime}$.

Обратимся теперь к диаграмме максимумов (см. рис.3) и воспользуемся условием $A B<B C<C A$. Из $A B<C A$ легко следует $\angle I_{a} B C<\angle I_{a} C B, \quad \angle B I_{a} I<\angle C I_{a} I$, откуда получаем, что высота треугольника $I_{a} B C$, проведенная из $I_{a}$, находится внутри угла $I I_{a} C$. Аналогично, из $A B<B C$ следует, что высота треугольника $I_{C} A C$, проведенная из $I_{c}$, находится внутри угла $I I_{b} C$; а из $B C<C A$ следует, что высота треугольника $I_{b} B C$, проведенная из $I_{b}$, находится внутри угла $I I_{c} A$. Значит, точка $O^{\prime}$ лежит внутри углов $I I_{a} I_{b}, I I_{b} I_{a}$ и $I I_{c} I_{b}$, т.е. находится внутри треугольника $I I_{b} C$. Видим, что этот треугольник состоит из синей и красной областей, границей между которыми является дуга окружности $\left(I_{a} I_{c} A C\right)$. Если точка $O^{\prime}$ находится внутри этой окружности, т.е. в синей области, то $\angle I_{a} O^{\prime} I_{c}>90^{\circ}$, откуда $\angle I_{a} I_{b} I_{c}>45^{\circ}$, что соответствует условию $\angle A B C<90^{\circ}$, значит, при $\angle A B C<90^{\circ}$ максимальный педальный отрезок - это $X_{b} Y_{b}$. При $\angle A B C>90^{\circ}$ точка $O^{\prime}$ лежит в красной области, значит, максимальный педальный отрезок $-X_{c} Y_{c}$. При $\angle A B C=90^{\circ}$ имеем $X_{b} Y_{b}=X_{c} Y_{c}>X_{a} Y_{a}$.

Мы получили полный ответ, и вроде бы задача М2621 решена. Однако в формулировке задачи есть тонкость: обратим внимание, что в условии сказано, что данная педальная окружность пересекает стороны (а не их продолжения). Во всех наших рассуждениях выше расположение проекций $X_{a}, Y_{a}$ и т.д. никак не использовалось.
Оказывается, при $\angle A B C \geq 90^{\circ}$ наша педальная окружность точки $O^{\prime}$ содержит вершину $B$ внутри себя, значит, в этом случае педальные отрезки, лежащие на прямых $A B$ и $B C$, не могут лежать на отрезках $A B$ и $B C$. Докажем это.

Точка касания $X_{a}=A_{1}$ такова, что

$$
C X_{a}=\frac{A B+B C-A C}{2}<\frac{A B}{2},
$$

поэтому $A_{1}$ лежит на отрезке $C A_{0}$, где $A_{0}-$ середина $B C$ (рис.5). Аналогично, точка

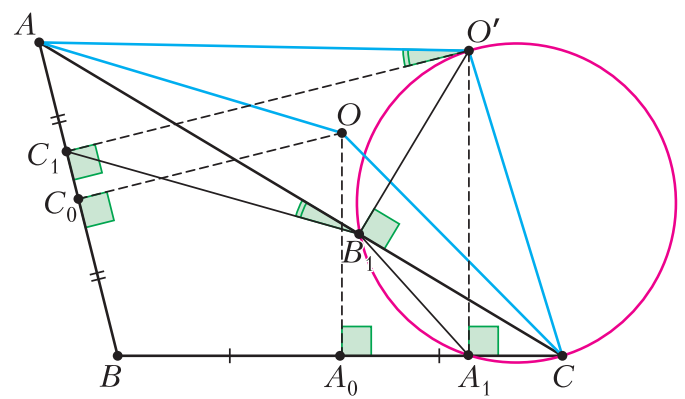

Pис. 5

$C_{1}$ отрезка $A B$ лежит на отрезке $A C_{0}$, где $C_{0}$ - середина $A B$. В силу условия $\angle A B C \geq 90^{\circ}$, точка $O$ пересечения серединных перпендикуляров к $A B$ и $B C$ лежит по другую сторону от $B$ относительно прямой $A C$, а значит, точка $O^{\prime}$ - тоже, при этом $O$ лежит внутри треугольника $A O^{\prime} C$. Заметим, что четырехугольник $O^{\prime} B_{1} A_{1} C$ - вписанный (в окружность с диаметром $O^{\prime} C$ ), откуда $\angle A_{1} B_{1} C=\angle A_{1} O^{\prime} C=90^{\circ}-\angle A_{1} C O^{\prime}<$ $<90^{\circ}-\angle A_{1} C O=\angle A_{1} O C=\angle B A C$. Аналогично, $\angle C_{1} B_{1} A<\angle B C A$.

Получаем, что $\angle A_{1} B_{1} C_{1}=180^{\circ}-\left(\angle A_{1} B_{1} C+\right.$ $\left.+\angle C_{1} B_{1} A\right)>180^{\circ}-(\angle B A C+\angle B C A)=\angle A B C$. Тогда $\angle A_{1} B_{1} C_{1}+\angle A_{1} B C_{1}>90^{\circ}+90^{\circ}=180^{\circ}$. Значит, $B$ лежит вне окружности $\left(A_{1} B_{1} C_{1}\right)$, что мы и хотели доказать.

\section{Еще несколько фактов}

В завершение приведем еще несколько задач, в которых сформулированы интересные факты для остроугольного треугольника $A B C$ на ту же тему. Докажите их.

1. Как известно, окружность девяти точек является педальной для ортоцентра $H$ 
или центра описанной окружности $О$. Если средний угол треугольника меньше $60^{\circ}$, то наименьший из педальных отрезков для $X=H$ лежит на наименьшей стороне, если же средний угол треугольника больше $60^{\circ}$, то - на наибольшей стороне.

2. Пусть $r$ - радиус вписанной окружности, $r_{b}-$ радиус вневписанной окружности, касающейся средней стороны, $R$ - радиус описанной окружности. Тогда наименьший из педальных отрезков задачи М2621 (хорд окружности Нагеля) лежит на меньшей стороне если и только если $2 R<r+r_{b}$.
3. Наибольшая из хорд педальной окружности точки Торричелли лежит на средней стороне треугольника.

4. Наименьшая из хорд педальной окружности точки Торричелли лежит на меньшей стороне, если средний угол треугольника больше $60^{\circ}$, и на большей стороне - в противном случае.

Можно продолжить исследование для других замечательных точек треугольника, а также для случая тупоугольного треугольника.

\section{Скрытая симметрия}

\section{E. БAKAEB}

В этом номере приводится решение задачи M2644. Красота этой задачи в том, что, несмотря на совершенно разные правила хода для двух игроков, оказывается, можно взглянуть на ситуацию под таким углом, что в качестве стратегии работает симметрия непосредственно в геометрическом смысле!

Мы приведем еще три игры с похожей «изюминкой». В задачах 1 и 2 игроки ходят по одинаковым правилам, но все же положение фигур на доске симметрией не обладает - предлагаем подумать, как этой симметрии достичь. В задаче 3 и вовсе игроки ходят совершенно по-разному, даже никакого расположения предметов не задается, но и там, тем не менее, разглядев скрытую симметрию, вы решите задачу.

1 (А.Толпыго). Белая ладья стоит на поле b2 шахматной доски $8 \times 8$, а черная на поле с4. Игроки ходят по очереди, каждый своей ладьей, начинают белые. Запрещается ставить свою ладью под бой другой ладьи, а также на поле, где уже побывала какая-нибудь ладья. Тот, кто не может сделать ход, проигрывает. Кто из

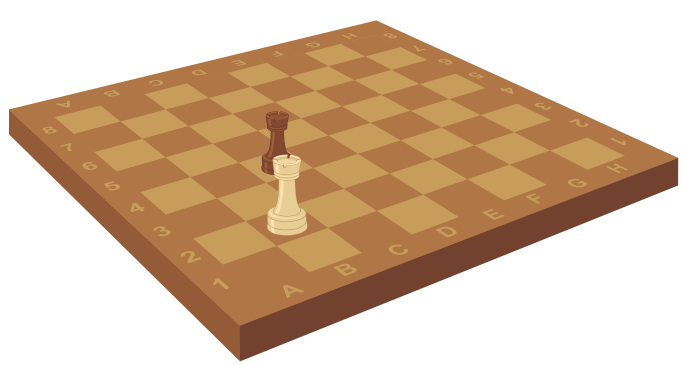

игроков может обеспечить себе победу, как бы ни играл другой? (За ход ладья сдвигается по горизонтали или вертикали на любое число клеток, и считается, что она побывала только в начальной и конечной клетках этого хода.)

2 (А. Бучин, А. Иванищук). Двое игроков по очереди выставляют на доску $65 \times 65$ по одной шашке. При этом ни в одной линии (горизонтали или вертикали) не должно быть больше двух шашек. Кто не может сделать ход - проиграл. Кто выигрывает при правильной игре?

3 (Е.Бакаев). Петя и Вася играют в такую игру. Сначала на столе лежит 11 кучек по 10 камней. Игроки ходят по очереди, начинает Петя. Каждым ходом игрок берет 1, 2 или 3 камня, но Петя каждый раз выбирает все камни из любой одной кучи, а Вася всегда выбирает все камни из разных кучек (если их больше одного). Проигрывает тот, кто не может сделать ход. Кто из игроков может обеспечить себе победу, как бы ни играл его соперник? 